\section{¿ES POSIBLE DELIBERAR MÁS ALLÁ DE LA OPINIÓN EN LOS COMITÉS DE BIOÉTICA? UNA RESPUESTA ACTUAL A PARTIR DE LA REFLEXIÓN EN TORNO A LOS AUTORES CLÁSICOS}

\author{
Juan-Pablo Faúndez-Allier ${ }^{1}$ \\ Pontificia Universidad Católica de Valparaíso, Chile \\ juan.faundez@ucv.cl
}

\section{IS IT POSSIBLE TO DELIBERATE BEYOND INDIVIDUAL OPINIONS WITHIN BIOETHICS COMMITTEES? \\ A CURRENT RESPONSE BASED ON REFLECTION ON THE CLASSIC AUTHORS}

\begin{abstract}
Cómo citar este artículo/Citation: Faúndez-Allier, J.P. (2014). “¿Es posible deliberar más allá de la opinión en los Comités de Bioética? Una respuesta actual a partir de la reflexión en torno a los autores clásicos". Arbor, 190 (766): a124. doi: http://dx.doi.org/10.3989/arbor.2014.766n2016
\end{abstract}

Recibido: 18 abril 2013. Aceptado: 9 diciembre 2013.

RESUMEN: A partir de un excursus histórico que pretende describir la definición de la práctica deliberativa, este artículo trata la deliberación como medio de resolución procedimental al interior de los Comités de Bioética de los hospitales. Lo peculiar del estudio consiste en que el autor argumenta que dada la conformación de los miembros del Comité, como especialistas en diversos ámbitos, las conclusiones a las que estos pueden llegar, actualmente, superan las meras opiniones, mediante deliberaciones cualificadas.

PALABRAS CLAVE: Comités de Bioética; deliberación; opinión; fundamentación.
Copyright: @ 2014 CSIC. Este es un artículo de acceso abierto distribuido bajo los términos de la licencia Creative Commons Attribution-Non Commercial (by-nc) Spain 3.0.

ABSTRACT: Starting with a historical excursus seeking to describe the definition of deliberative practice, this article treats deliberation as a form of procedural resolution within hospital Bioethics Committees. What is special about this study is that the author argues that, given the way in which the Committee is made up of specialists in diverse areas, the conclusions they reach through qualified deliberations can transcend mere opinion.

KEYWORDS: Bioethics Committees; deliberation; opinion; foundation. 


\section{INTRODUCCIÓN}

El ejercicio de la deliberación es de antigua data en la historia de la Humanidad. Resabios de sus orígenes están recogidos en la República de Platón, texto en el que el discípulo de Sócrates hace explícita la mención en orden a entender esta práctica de forma diversa a como la concibiera su maestro Sócrates. Para Platón, la aplicación deliberativa debía seguir una derivación deductiva de las ideas puras para ser ejercidas en la práctica. A diferencia de él, mediante los élenkhos, Sócrates propone criterios de razonabilidad en una línea no deductiva, sino probable y para ser aplicados al caso, esbozando un criterio más cercano al que empleará Aristóteles en la Ética a Nicómaco. Junto a esta obra, ya en el Libro I de la Retórica, hablando de los tres modos en que puede desarrollarse un discurso retórico, el Estagirita se referirá en primer lugar al deliberativo -además del judicial y el epidíctico-, explicitando que es el que mediante el consejo y la capacidad de convencimiento va desde la oportunidad de aportar elementos del discurso, en el ámbito privado, para conseguir la disuasión de quienes escucharán en una esfera de discusión más abierta (Aristóteles, 1990, 1358b-1359a). Yendo más allá, una clara proyección y explicitación del ejercicio deliberativo es el que realiza Aristóteles en el Libro IV de la Política, texto en el que se refiere a la aplicación de este modo de proceder en los distintos tipos de configuración cívica (Aristóteles, 2000, 1298a-1299a).

Durante el siglo $X X$, se ha puesto de relieve la necesidad por enriquecer los criterios deliberativos que, curiosamente, se han mantenido por siglos sin ser explícitamente desarrollados más allá de la riqueza argumentativa que aportaron los análisis de autores clásicos. De este modo, Ezequiel y Linda Emanuel, entre otros, redescubrirán en estos días el método deliberativo como el modelo ético más excelente para la práctica médica, dado que promueve las características del que llaman médico humanista. Este es quien mediante un diálogo persuasivo ha de ayudar a incorporar la actividad deliberativa en las reflexiones que los usuarios del sistema de salud han de realizar en vistas al aporte autónomo con el que puede contribuir quien demanda la prestación de un servicio sanitario; una necesaria disposición integrativa en las relaciones contemporáneas entre médico y "paciente" (Emanuel y Emanuel, 1999, 123s.).

Uno de los espacios donde esta práctica vuelve a cobrar especial importancia es el que se da en los Comités de Ética Asistencial (CEA) de los hospitales, instancias en las que mediante el ejercicio de la prudencia y la reflexión razonable, un equipo interdisciplinario está llamado a sugerir propuestas de resolución para casos especialmente delicados. Pero cabe plantearse si a lo que se estaría apuntando actualmente como proceso deliberativo en la práctica médica corresponde exactamente a lo que entendían por el mismo los antiguos autores que se refirieron a este tema. Surge entonces una pregunta que podríamos formular en orden a distinguir a qué nos debiéramos referir cuando hablamos de procesos de deliberación en los centros de salud contemporáneos, y cuál será el objeto al que actualmente debieran tender: ¿Habrá algún indicio para considerar que al interior de los CEA la deliberación que se da pueda superar el mero intercambio de opiniones con ánimo persuasivo?

La tarea del presente artículo irá tras la búsqueda de distinciones, en esta línea, para intentar esclarecer a qué nos estamos refiriendo actualmente al hablar de deliberación en la práctica médica y cómo se podría mostrar la especificidad de esta metodología en el contexto contemporáneo.

\section{HACIA LA DELIBERACIÓN EN LA PRÁCTICA DE LOS COMITÉS DE BIOÉTICA}

La práctica creciente y cada vez más compleja de los CEA ha llevado a justificar filosóficamente su praxis. Una vez que la bioética se fuera asentando como incipiente disciplina, en la década de los setenta ${ }^{2}$, surge prontamente la necesidad de configurar el camino que ayudaría al razonamiento moral a seguir un procedimiento formal, buscando con ello resolver las problemáticas que cada vez se imponían con mayor gravedad y frecuencia. Se hacía imprescindible, de esta forma, articular la debida confluencia que llamaba a relacionar satisfactoriamente los hechos clínicos junto con los elementos de análisis ético, superando las experiencias anteriores que marcaban una irreconciliable separación entre ambas posturas. La bioética parecía como un nuevo escenario de disputa entre filósofos y médicos, siendo considerada por los primeros como una modalidad de la ética aplicada en la que los clínicos debían aportar sólo la materia de investigación, en tanto que los filósofos aplicarían una serie de principios acompañados de un debido procedimiento (Gracia, 1991). Para los segundos, se trataba de un nuevo campo de decisión que necesariamente debía ser iluminado por la actividad clínica, y en el que los filósofos no tenían mucho que decir. No obstante, con el correr de los años, fue posible constatar, por ambos sectores, que la aplicación de los famosos cuatro principios de la disciplina - no-maleficencia, jus- 
ticia, autonomía y beneficencia-, por si sola, no lograba resultados favorables, toda vez que la toma de decisiones dependía del devenir argumentativo y del contexto que surgía a partir del caso concreto (Cfr. Álvarez, 2005). Al mismo tiempo, el intento de los médicos por reducir los problemas morales a meras situaciones técnicas tampoco era sostenible, dado que se dejaba de lado la apreciación de los valores con que era necesario iluminar de contenido moral las decisiones que se tomaran (Ferrer y Álvarez, 2003). Surgía así un presupuesto que habría de tenerse en cuenta, y que evidenciaría la necesaria conjunción entre las actividades médica y filosófica, a través de la vía deliberativa (Gracia, 2001, 2008, 2011a, 2011b).

Para Platón, la deliberación es un tipo de conocimiento que surge desde la deducción de los principios morales necesarios para las situaciones de la vida concreta, siendo concebida como una metodología de aplicación de la Teoría de las Ideas. La deliberación en este caso tiene la función de definición o concreción de los principios en vistas de la situación fáctica. Por ello, a partir del texto de la República, la deliberación en la ética platónica toma el papel del método por el que se concretan los primeros principios. A partir del análisis de un pasaje clave de dicha obra (Platón, 1986, 428b-434d), Platón explica cómo conducir las ideas puras que se han conocido en virtud de la dialéctica al ámbito de la práctica, labor que debe ser realizada por los guardianes o políticos.

Desde otra perspectiva, en el contexto de análisis de las acciones voluntarias e involuntarias que Aristóteles lleva adelante en el Libro III de la Ética a Nicómaco, es posible ver plasmado un detallado estudio acerca de la deliberación en una aproximación diversa. En el tratamiento previo a esta forma de ejercer la toma de decisiones, el filósofo indaga acerca del alcance de la responsabilidad efectuando un estudio sobre la naturaleza de la elección. Esta última parece ser un elemento voluntario que se ejerce dentro de un ámbito específico que se diferencia de la realización de actos mediados por la fuerza o la ignorancia. La voluntariedad, desde una parte, señala que el principio del movimiento está en quien realiza la acción, por lo que depende de éste si aquella se lleva a cabo o no. Por eso las acciones forzosas o que carecen de voluntariedad tienen su causa fuera del agente, sin que éste pueda participar como ordenador de las mismas. En cuanto a la ignorancia, lo que se hace sin conocimiento de causa es descrito por Aristóteles como no voluntario; señalándose al mismo tiempo como involuntario aquello que se realiza dolorosamente o a causa de la obligación. Una vez hecha esta distinción, el Estagirita señala que se comprende por elección lo que configura la virtud y determina la caracterización de las acciones. Por ella se entiende algo distinto al impulso, al deseo, o la opinión. El fundamento de la elección estaría en la inclinación razonable hacia los medios, entendidos como posibilidad efectiva y real de alcanzar un fin, mediante el análisis de los elementos que lo acompañan. Con ello sobrepasaría la posibilidad del impulso y el deseo. Pero además superaría la opinión, que es aquel pronunciamiento que se otorga sin investigar el objeto y que se señala en relación a la falsedad o la corrección relativa. A diferencia de la opinión, la elección se determina estimando la bondad o la maldad de un acto. Se elige, por tanto, lo que se considera bueno, y se opina acerca de lo que no se reconoce claramente como tal.

\section{LA APLICACIÓN DE LA DELIBERACIÓN EN LOS COMITÉS DE ÉTICA ASISTENCIAL: ¿UN MÉTODO PARA OBTENER MERAS OPINIONES?}

En el contexto de esta distinción cabe, entonces, hablar de deliberación, entendida como la indagación sobre lo que está al alcance y es posible de realizarse, aunque no esté aclarada perfectamente la catalogación en el sentido de si consiste en algo bueno o malo. Como señala Aristóteles, lo que se busca en todo acto es el bien o la rectitud, aunque ésta pueda comprender formas que no encaminan con claridad al propio bien, desde el momento en que puede perseguirse razonablemente un fin malo. Lo opuesto puede ocurrir también efectuando un razonamiento que se inicie desde términos falsos hasta llegar a obtener un fin bueno. El fin bueno que se obtendría, aun cuando pudiera alcanzarse a partir de premisas falsas, no lograría la consideración de bondad en la perspectiva moral: "Pero también es posible alcanzarlo - el bien - mediante un razonamiento falso, y alcanzar lo que se debe hacer, pero no por los medios debidos, sino por un término medio falso; de modo que no será buena deliberación ésta en virtud de la cual se alcanza ciertamente lo que se debe, pero no por el camino debido" (Aristóteles, 1989, 1142b 26-30). En el fondo, se trata de la consagración por la que se establece que desde el punto de vista moral el fin no justifica los medios, aunque lógicamente esto pueda ser posible.

Del mismo modo, acerca de lo que puede acontecer más allá de la influencia humana no cabe hablar de deliberación, dado que ya no se trata de una posibilidad que se mueve en la esfera de la contingencia. Por tanto, la deliberación se emplea para referirse a un 
modo de investigar, indagar o calcular, vinculado con lo que usualmente acontece en la esfera de las decisiones humanas. Es, entonces, un pronunciamiento fruto de la investigación, de carácter contingente, sin que se diga expresamente que ello consista en una opinión, sin tratarse al mismo tiempo de ciencia:

Tampoco consiste la buena deliberación en ninguna clase de opinión. Pero puesto que el que delibera mal yerra y el que delibera bien lo hace rectamente, es claro que la buena deliberación consiste en una especie de rectitud, que no es propia ni de la ciencia ni de la opinión [...]. Luego tiene que consistir en la rectitud del discurso; ésta, en efecto, no es todavía afirmación, y mientras que la opinión no es indagación, sino ya una especie de afirmación, el que delibera, tanto si delibera bien como si lo hace mal, indaga y calcula (Aristóteles, 1989, 1142 b 11-14. 16-19) ${ }^{3}$.

Un argumento que ya en la Antigüedad parecía marcar una diferencia clara entre la deliberación propiamente tal y la mera capacidad de verter opiniones. Para Diego Gracia, quizás el más importante bioeticista de Iberoamérica, en sus análisis acerca del alcance de la comprensión de la actividad deliberativa aristotélica como proceder metodológico de la bioética, la estimación de la deliberación -entendida como una forma de organizar la mera opinión basada en un acto de razonamiento en el que pareciera no intervenir el cálculo y la indagación en torno a los medios- es lo que especifica esta actividad (Gracia, 2008, 9): “Este es el mundo propio de la 'opinión'. Cuando opinamos damos razones de algo, razones que consideramos de peso, pero no de tanto peso que nieguen la posibilidad de otras razones alternativas. Cuando uno opina no niega la posibilidad de opiniones distintas e incluso contrarias" (Gracia, 2001, 31). Para este autor, efectivamente, se podría mencionar la deliberación como un procedimiento que se articula en base a meras opiniones, al consistir en un asunto que no estaría perfectamente definido como bueno o malo. No obstante, uno de los requisitos establecidos por el Estagirita para deliberar es el de realizar previamente una tarea investigativa o de indagación que vaya más allá de un mero pronunciamiento en el que no se haya concretado esta etapa.

Aun cuando, como pretendo demostrar, si bien es cierto que la deliberación no se relaciona con la epistéme, al no consistir en conocimiento exacto, tiene una pretensión que va más allá de la mera dilucidación de para-doxas, o de opiniones - dóxas- que se contraponen sin ninguna consideración indagatoria. Esto es lo que quiere hacer resaltar Aristóteles, desde el momento en que sostiene que aunque el discurso no sea cierto o apodíctico, en el caso de la deliberación, eso no significa que se trate de opinión, puesto que consiste en una afirmación que se sostiene en una investigación preparatoria, moviéndose la opinión sólo en el ámbito de las afirmaciones carentes de indagación e investigación previas. Y, justamente, aplicando esta argumentación al ámbito de las decisiones que se obtienen en la esfera de los CEA, me parece que la constitución efectiva de los mismos, aun sin quererlo, apunta en el sentido de la necesidad de la investigación preparatoria. Lo señalo ya que si no fuera relevante la preparación formativa que han desarrollado los miembros de los Comités, que precede a las consultas que se les formulan, no tendría sentido que la conformación de los mismos reúna a profesionales preparados de distintas áreas con el objeto de tomar una decisión en la que se necesite manejar conocimientos específicos de diversa procedencia: médicos, filosóficos, científicos, jurídicos o psicológicos (Ferrer y Álvarez, 2003).

De entrada, podríamos considerar que no se trata de que los especialistas en sus materias adopten una actitud incoherente en cada sesión, dejando de lado sus conocimientos adquiridos para asumir el papel de quienes emiten una mera opinión para el caso concreto, sino que aportan a la tarea deliberativa justamente sus conocimientos y su experiencia específica, mediante los cuales se construirán una o diversas decisiones colectivas, quizá distintas a las que cada cual podría haber defendido por separado, pero razonando en base a ciertos conocimientos específicos que se ofrecen de modo colectivo. Por ello, los encargados de deliberar con el objeto de conseguir una respuesta no vinculante son especialistas que comparten una reflexión encaminada a ofrecer un planteamiento que presupone conocimientos concretos. Estos son los que justifican la presencia y la labor de los integrantes del Comité, y lo que fundamenta sus comentarios de cara a la decisión. Un objetivo final que aunque no desemboque en una conclusión científica, o estrictamente necesaria, se puede clasificar como parte de una categoría distinta a la mera opinión, en función de la formación específica de quienes formulan una respuesta que pretende dar luces sobre un determinado problema, que se establece a partir de criterios investigativos que se obtienen con anterioridad. En definitiva, el criterio con el que se zanjan las decisiones de los CEA está influenciado por el análisis de los hechos a la luz de los estudios médicos, científicos, éticos o jurídicos que determinarán ciertas líneas de respuesta, buscándose con ello vías aclaratorias que pretenden explicitar los conocimientos y las investiga- 
ciones previas de sus miembros. En caso contrario, lo que debiera acontecer para garantizar que lo que se dé sean simples opiniones, sería convocar a un conjunto de personas escogidas al azar y sin preparación para manifestar sus apreciaciones de modo razonado, careciendo de los conocimientos específicos y las habilidades idóneas, elementos fundamentales en la actividad deliberativa. Por tanto, la expresión deliberativa que se percibe al interior de los CEA es la de una metodología resolutiva que se cualifica en función del manejo a fronte y a tergo de los mismos conocimientos, que se exponen en un contexto de discusión que está influido y, de alguna forma, delimitado por las propias competencias de los miembros del Comité.

\section{PROFUNDIZANDO EN LA FUNDAMENTACIÓN DELIBERATIVA MEDIANTE LA VÍA ARISTOTÉLICA}

En el libro VI de la Ética a Nicómaco, el Estagirita va a profundizar en el ejercicio de la deliberación partiendo de la explicitación del sentido de las virtudes intelectuales: aquellas disposiciones que permiten conseguir la verdad, como fin propio del intelecto. Habrá en este sentido virtudes intelectuales relacionadas con el entendimiento especulativo -inteligencia, ciencia y sabiduría - y virtudes intelectuales vinculadas con el entendimiento práctico - arte y prudencia. La prudencia (phrónesis), aquel hábito que especifica qué se debe hacer en cada caso, tiene una posición no exclusiva dentro de este cuadro, ya que puede ser considerada, a la vez, como una virtud moral que señala lo adecuado en cada situación, con el objeto de conseguir el bien. Por otro lado, las virtudes morales son aquellas que, adquiridas mediante la práctica reiterada de ciertos hábitos, disponen concretamente para la realización de la vida buena. Es lo que efectúan mediante la perfección de la voluntad - gracias a la virtud de la justicia- y la correcta dirección de las potencias apetitivas inferiores - concupiscible e irascible- que se relacionan con las pasiones, encaminadas por las virtudes de la templanza y la fortaleza. Por ello, la prudencia, además de tener un lugar entre las virtudes intelectuales, se identifica entre las virtudes morales. De ahí que Aristóteles la mencione como un modo de ser racional verdadero y práctico - los fines propios de las virtudes intelectuales - en orden a precisar lo bueno y lo malo para el ser humano -el ámbito de discernimiento propio de las virtudes morales. Así, el Estagirita concebirá a la prudencia como la "disposición racional verdadera y práctica respecto de lo que es bueno y malo para el hombre" (Aristóteles, 1989, 1140b 6-8). Añadiendo, en este mismo sentido, que la prudencia es "un modo de ser racional, verda- dero y práctico, respecto de lo que es bueno para el hombre" (Aristóteles, 1989, 1140b 20-22).

La prudencia, por tanto, es la disposición que busca racionalmente lo que de forma verdadera y práctica es bueno para el ser humano. Si lo que se busca es el bien que ya se ha definido, y esto se hace de modo racional y verdadero - auxiliado por las restantes virtudes dianoéticas-, parece que se está señalando algo que va más allá de la mera opinión. En este sentido, Aristóteles señala que "la buena deliberación consiste en una especie de rectitud, que no es propia ni de la ciencia ni de la opinión" (Aristóteles, 1989, 1142b 1314), un modo de razonar que sin ser científico aporta algo más que la mera dóxa, explicitando una pretensión de mayor precisión.

El factor problemático por el que se plantea la deliberación como una realidad distinta a la dilucidación plenamente certera, consiste en que el ejercicio deliberativo no razona a través de una única vía de análisis, sino en un contexto pluralista de caminos que se abren de forma diversa. De ahí que Aubenque sostenga en su análisis de la deliberación aristotélica que las opciones que se vislumbran en este sentido no darían más cabida que la mera opinión, lo que precisamente ocurre en contextos como el de la medicina (Aubenque, 1999, 127). Por ello comenta este autor, en alusión a la Retórica de Aristóteles, que en un cuadro de proyección temporal, el género deliberativo no es el que corresponde a la esfera de la decisión judicial que aborda hechos que atañen al pasado-, ni al género de las alabanzas discursivas - propio del presente-, sino a propuestas futuras, por tanto, probables y dadas en un escenario de proyección prospectivo; momento temporal que justamente debe abordar el CEA en la esfera de sus pronunciamientos.

La recta razón apunta a la aplicación del justo medio en relación a las virtudes morales, tomando como base ciertos principios desde los cuales lleva adelante su actividad silogística. De este modo, se puede comprender la phrónesis, prudencia o recta razón de lo agible, desde el punto de vista de los principios. Y detengámonos en lo siguiente: si bien es cierto que con la recta razón se apunta a una disposición universal que manda deontológicamente de forma principial o normativa, lo que debiera hacerse de forma correcta; con la mención a lo agible se está refiriendo al mismo tiempo a una situación particular de orden teleológico, o en función de las consecuencias - la alternativa buena o menos mala que se obtiene en vista de las excepciones a la regla-, con motivo de prevenir los efectos del acto. De este modo, podemos inter- 
pretar a Aristóteles colocándolo en una disposición deontológico-teleológica que plantea la deliberación como una praxis que puede ir más allá de la simple opinión carente de la necesaria investigación. Es decir, se trataría, en este caso, de instancias del proceso que contribuyen en la búsqueda de una decisión verdaderamente prudente y poseedora de gran resguardo intelectual, y no de meras opiniones que carecen de estos presupuestos. Ya que la deliberación se aplica en un ámbito que no aspira a una deducción de carácter necesario, se debe buscar para la resolución de los casos que la requieran, el consejo práctico que sea más razonable. Así, la respuesta lleva a la ponderación de los medios que conducen a la obtención del fin, retrotrayendo la secuencia hasta la causa primera. Una forma específica de indagación razonada que, desde la deliberación en torno a los fines, pretende justificar los medios que encaminan hacia la realización de las actuaciones.

Por ello, la práctica deliberativa puede ser entendida como una forma de profundización e investigación que se preocupa de los medios para alcanzar un fin específico de forma prudente, ponderando hasta dónde sea posible los elementos deontológicos y teleológicos que están en juego. De este modo, el objeto de la deliberación es el medio que permite la obtención de la finalidad. Este objeto es semejante para la elección, ya que finalmente se elige aquello que se ha tomado como fruto de la actividad deliberativa. Así, el fin bueno es el objeto de la voluntad, mientras que los medios de la misma corresponden a la elección y la deliberación, con la diferencia que ya he mencionado. Los medios operan a través del ejercicio de las virtudes y la superación de los vicios, que son hábitos operativos que siempre están al alcance, ya sea de la realización del bien o de su no concreción. Es por ello que la virtud moral tiene por objeto la elección del término medio entre dos vicios opuestos. El ejercicio de una $u$ otra cosa, o negarse a la realización del mismo, es lo que irá determinando la virtuosidad o la vida inclinada al vicio. Y, lo que va definiendo la inclinación en uno $u$ otro sentido, es la práctica reiterada de actos que encaminen en este sentido. Reitero, considerando que la prudencia es un hábito que tanto en el ámbito de las virtudes dianoéticas como de las éticas lleva a un tipo de resultado que supera lo meramente opinable.

\section{CONCLUSIÓN}

En definitiva, en este itinerario deliberativo, en relación al contexto problemático y de avance histórico en el que se mueve la experiencia humana, tenemos siempre delante la dimensión proyectiva por la que el ser humano se abre en oportunidades y alternativas con algún grado de certeza, que es justamente la que se requiere en el ejercicio deliberativo cualificado - y no de la mera opinión- que desarrollan los miembros de los CEA al dar una respuesta. La deliberación es la vía metodológica que permite ir sorteando los desacuerdos en contextos de resolución que deben avanzar en la superación de conflictos mediante un aseguramiento deontológico-teleológico de rasgos intersubjetivos. Así, el nuevo escenario que se vislumbra para llevar a cabo un aterrizaje práctico de estos temas, es el que se abre en bioética a través de este proceder inclusivo y universalizador que permite la deliberación, con las precisiones que he señalado. La relación a fronte y a tergo marca el paso desde el momento en el que se sostiene la argumentación en base a principios o valores que asumen un carácter deontológico, y las circunstancias y consecuencias que se aprecian como el componente teleológico. Por ello se puede hablar, en el ejercicio que desarrollan Ios CEA, de una praxis deliberativa que posee el atributo de la necesaria investigación previa. La indagación que determina lo conveniente en base a principios, valores, circunstancias y consecuencias, que superan en su articulación el mero establecimiento de una opinión razonable.

Ciertamente, la pretensión de proporcionar una respuesta por parte de los CEA a quienes requieren su pronunciamiento, no resulta completamente certera al modo de una aseveración de tipo científico. No obstante, uno de los requisitos demostrativos que persigue la reconstrucción sociolingüística de la ética, en el contexto contemporáneo, persigue constatar, mediante una vía discursiva de carácter procedimental, la posibilidad de establecer criterios de validez ampliamente reconocidos, mediante la vía del diálogo intersubjetivo. El acuerdo de los sujetos que dialogan, posibilitado por la competencia de los interlocutores, es lo que permite superar los atisbos de la subjetividad autónoma. Como dirá Apel, en La transformación de la filosofía, el argumento intersubjetivo mediado por los signos de interrelación adecuados es el que permite lograr acuerdos en la comunidad ideal de comunicación con carácter de verdad y sentido. De hecho, el objetivo que se plantea en este contexto, es el de la superación del relativismo mediante una estructura performativa sostenida por el lenguaje. Por ello, desde la perspectiva habermasiana, la acción comunicativa basada en el consenso adquiere valor de verdad en la medida en que la mediación lingüística es capaz de traspasar los valores morales de forma inter- 
subjetiva. La vida social es posible gracias al mínimo de valores aceptados por todos mediante el diálogo y el consenso. Así, los CEA se constituyen, según mi parecer, queriendo incorporar, justamente, los crite- rios de una comunidad ideal de comunicación que en cada sesión pretende concretar sus procedimientos para dar forma a una comunidad real de deliberación.

\section{NOTAS}

1 Abogado y Licenciado en Filosofía. Más ter en Derecho. Doctor en Filosofía (premio extraordinario de doctorado por la Universidad de Salamanca 2011-2012). Profesor de Filosofía Moral, Derecho Canónico y Jefe de Carrera de la Facultad de Teología en la Pontificia Universidad Católica de Valparaíso, Chile.
2 Cuyos analistas no tenían a la vista en ese entonces los tratamientos que hiciera en la década de los años veinte el filósofo y teólogo alemán Fritz Jahr, quien podría ser considerado el verdadero 'padre' de la disciplina (Cfr. Lolas, 2008a, 2008b).
3 Según lo que señala Aristóteles, la opinión es sólo una especie de afirmación que no ha tenido una base investigativa previa, lo que en este caso difiere de la deliberación. A la opinión le falta este último elemento por lo que, a mi juicio, no sería indicado considerar la actividad deliberativa como una que se mueve, sin más, en este contexto. Por ello, la deliberación es algo distinto a la ciencia y la opinión.

\section{BIBLIOGRAFÍA}

Álvarez, Juan Carlos (2005): “Procedimiento y metodología de la decisión", en ibidem, Principios y aplicaciones de la Bioética (I Congreso Interdisciplinar de la Asociación de Bioética Fundamental y Clínica), Madrid, Asociación de Bioética Fundamental y Clínica, pp. 131-151.

Aristóteles (1989): Ética a Nicómaco (traducción de María Araujo y Julián Marías), Madrid, Centro de Estudios Constitucionales.

Aristóteles (1990): Retórica (traducción, prologo y notas de Antonio Tovar), Madrid, Centro de Estudios Constitucionales.

Aristóteles (2000): Política (traducción y notas de Manuela García Valdés), Madrid, Gredos.

Aubenque, Pierre (1999): La prudencia en Aristóteles, Barcelona, Crítica.

Emanuel, Ezequiel y Emanuel, Linda (1999): "Cuatro modelos de la relación médicopaciente», en Couceiro, A., Bioética para clínicos, Madrid, Triacastela, pp. 109-126.
Ferrer, Jorge José y Álvarez, Juan Carlos (2003): Para fundamentar la bioética. Teorías y paradigmas teóricos en la bioética contemporánea, Madrid, Universidad Pontificia Comillas y Desclée de Brouwer.

Gracia, Diego (1991): "Hechos y valores en la práctica y en la ciencia médicas", en Lujosa, F. y otros, Actas del IX Congreso Nacional de Historia de la Medicina (septiembre de 1989), Vol. 1, Zaragoza, Secretariado de Publicaciones de la Universidad de Zaragoza, pp. 35-63.

Gracia, Diego (2001): “La deliberación moral", en Boletín de la Academia Chilena de Medicina, Santiago de Chile.

Gracia, Diego y equipo (2008): Guía para Comités de Ética Asistencial, Junta de Castilla y León, Consejería de Sanidad.

Gracia, Diego (2011a): “Deliberation and Consensus", en Chadwick, R. y otros [eds.], The SAGE Handbook of Healt Care Ethics, London, SAGE Publications, pp. 84-94.
Gracia, Diego (2011b): “Teoría y práctica de la deliberación moral”, en Feito, Lydia, Gracia, Diego y Sánchez, Miguel [eds.], Bioética: El estado de la cuestión, Madrid, Triacastela, pp. 101-154.

Lolas, Fernando (2008a): "Bioethics and Animal Research. A Personal Perspective and a Note on the Contribution of Fritz Jahr", in Biological Research 41, pp. 119-123.

Lolas, Fernando (2008b): "El "imperativo bioético" de Fritz Jahr y la neobioética estadounidense", en Jano. es: http://www.jano.es/jano/humanidades/medicas/fernando/lolas/ stepke/“imperativo/bioetico"/fritz/ jahr/neobioetica/estadounidense/_f303+iditem-3133+idtabla-4

Platón (1986): República, en Diálogos IV, (introducción, traducción y notas de Conrado Eggers), Madrid, Gredos. 